\title{
Numerical Study of Resistivity Scaling in $\pi$ Junction Granular Superconductors
}

\author{
Enzo Granato \\ Laboratório Associado de Sensores e Materiais, \\ Instituto Nacional de Pesquisas Espaciais, 12245-970 São José dos Campos, SP Brazil
}

Received on 23 May, 2003.

\begin{abstract}
Numerical simulations based on Monte Carlo dynamics are used to investigate the resistivity behavior of granular superconductors containing a random distribution of $\pi$ junctions, as in superconducting materials with $\mathrm{d}$-wave symmetry. The presence of $\pi$ junctions leads to quenched in circulating currents (chiralities) and to chiral glass behavior at low temperatures, even without an external magnetic field. An XY spin glass model in the phase representation is used to determine the current-voltage characteristics and critical exponents of the resistivity transition. In two dimensions, the linear resistivity is nonzero at finite temperatures and the dynamic scaling analysis of the nonlinear resistivity is consistent with a phase transition at zero temperature. In three dimensions, we find a transition at finite temperatures below which the linear resistivity vanishes and the corresponding critical exponents are determined from the scaling analysis. The results are in good agreement with Langevin simulations in the phase representation. The dynamic exponent $z$ is significantly different from previous results obtained in the vortex representation.
\end{abstract}

Granular superconductors with d-wave symmetry, as some of the unconventional high- $T_{c}$ superconducting materials, can be viewed as a network of Josephson junctions with frustration effects even in zero external magnetic field [1]. Frustration arises from the presence of $\pi$ junctions which introduce a phase shift of $\pi$ between superconducting regions, and to half-flux quantum vortices on closed loops with an odd number of these junctions [2, 3]. The magnetic properties of granular samples, arising from the orbital currents of theses vortices, have been extensively studied $[1,4,5,6,7]$ and provide an explanation of the paramagnetic Meissner effect [8]. Nevertheless, there are also important consequences for the resistivity behavior of theses systems which also deserve detailed investigations.

In a conventional granular superconductor, the phases of neighboring superconducting regions tend to be locked with zero phase shift, and a phase-coherence transition is expected for decreasing temperature into a superconducting state with vanishing linear resistivity. The critical behavior of this resistive transition is reasonable well understood both in the two dimensional limit and in three dimensions. On the other hand, for granular superconductors with a large concentration of $\pi$ junctions, frustration and disorder effects leads to a vortex glassy phase and the resistive behavior is much less understood. The simplest model of the system is to consider only contributions from the Josephson coupling energy of nearest-neighbor grains, $H_{i j}=-J_{o} \cos \left(\theta_{i}-\theta_{j}-t_{i j}\right)$, where $\theta_{i}$ is the phase of the local superconducting order parameter, $J_{o}>0$ and $t_{i j}=0$ or $\pi$ correspond to the phase shifts of 0 and $\pi$ junctions. This is equivalent to the interaction of two-component pseudo-spins $\vec{S}=(\cos (\theta), \sin (\theta))$, coupled by ferro or antiferromagnetic interactions, respectively, which leads to an XY-spin (chiral) glass model for the granular system [6]. The chiral variable can be defined as the direction of spin rotation in a frustrated loop and corresponds to the local circulating current (vortex) in a closed loop with and odd number of $\pi$ junctions. Based on Monte Carlo (MC) simulations [6, 7] for this model in three dimensions, it has been suggested that the equilibrium low-temperature state for the granular superconductor is a chiral glass but with no phase coherence and, therefore, the resistivity should be nonzero. This implies a chiral glass transition at a nonzero critical temperature but no resistive transition, except perhaps at zero temperature. Thus, strictly speaking, there is no true superconducting phase at low temperatures in this scenario. However, while different works agree on the existence of the proposed chiral glass transition, the situation regarding the resistive behavior in three dimensions is unsettled. Results for the ground state [9] of the XY-spin glass indicates that the lower critical dimension for phase ordering is between 2 and 3 and therefore a phasecoherence transition at finite temperatures is not possible in two dimensions but should occur in three dimensions. The critical temperature in three dimensions, however, can not be estimated from these calculations. In fact, numerical simulations of the resistive behavior in two dimensions shows that the transition occurs at zero temperature [10]. In three dimensions, dynamical simulations suggest a resistive transition at finite temperatures $[11,12]$. These simulations were based on different dynamics and different representations of 
the same XY-spin glass model. While the static exponents agree, as expected from the universality of critical behavior, the dynamic exponent $z \sim 4.6$ obtained from the resistivelyshunted-junction (RSJ) model of the dynamics in the phase representation [12] is significantly different from that obtained from MC dynamics, $z \sim 3.1$, in the vortex representation [11], suggesting a strong dependence of $z$ on the details of the dynamics. In view of the absence of precise agreement among these studies, additional numerical results using different dynamics are required to confirm the resistive behavior and determine the critical properties satisfactorily.

In this work, we study the resistivity behavior by a driven Monte Carlo dynamics simulation in the phase representation, both in two and three dimensions. The main advantage of this method, compared with previous Langevin dynamics simulations $[12,10]$, is that much longer time scales can accessed, insuring that the long-time behavior is probed at the lowest temperatures and current densities. The results show that, in two dimensions, the linear resistivity is nonzero at finite temperatures and the dynamic scaling analysis of the nonlinear resistivity is consistent with a phase transition at zero temperature. In three dimensions, we find a resistive transition at finite temperatures and the corresponding critical exponents are determined from the scaling analysis. The dynamic exponent $z$ is essentially the same as that obtained by Langevin dynamics. However, $z$ is significantly different from previous results obtained in the vortex representation by MC dynamics.

We consider granular superconductors with $\pi$ junctions, modelled by a three-dimensional XY-spin glass driven by an external current, described by the Hamiltonian

$$
H=-J_{o} \sum_{<i j>} \cos \left(\theta_{i}-\theta_{j}-t_{i j}\right)-J \sum_{i}\left(\theta_{i}-\theta_{i+x}\right)
$$

The first term gives the nearest-neighbor Josephson coupling energy, where the quenched phase shift $t_{i j}$ is equal to $\pi$ or 0 with equal probabilities, corresponding to the standard $\pm J_{o}$ $\mathrm{XY}$ spin glass model $[6,7,9,10,12,11]$. The second term in Eq. 1 represents the effects of an external driving current on the superconductor, applied in the $x$-direction. When $J \neq 0$, the total energy is unbounded and the system is out of equilibrium. The lower energy minima occur at increasing phase differences $\theta_{i}-\theta_{i+x}$ as a function of time, leading to a net voltage $V$ proportional to the phase slippage $<d\left(\theta_{i}-\theta_{i+x}\right) / d t>$.

To study the nonequilibrium behavior generated by the driving current density $J$ in Eq. 1, we use a driven MC dynamics method. The time dependence is obtained by identifying the MC time as the real time $t$ and we take the unit of time $d t=1$, corresponding to a complete MC pass through the lattice. Periodic (fluctuating twist) boundary conditions are used [13] in square and cubic systems of linear size $L$. This boundary condition adds new dynamical variables, $u_{\alpha}$ ( $\alpha=x, y$ and $z$ ), corresponding to a uniform phase twist between nearest-neighbor sites along the principal axis directions $\hat{x}, \hat{y}$ and $\hat{z}$. A MC step consists of an attempt to change the local phase $\theta_{i}$ and the phase twists $u_{\alpha}$ by fixed amounts, using the Metropolis algorithm. The external current density $J$ in Eq. 1 biases these changes, leading to a net phase slippage across the system, $d\left(\theta_{1}-\theta_{L}-u_{x} L\right) / d t$, which is used as a measure of the voltage in arbitrary units. Using this procedure, the voltage $V$ (electric field $E=V / L$ ) was computed as a function of the driving current density $J$ for different temperatures.

The nonlinear resistivity $E / J$ as a function of current density $J$ and temperature $T$ for the two-dimensional case is shown in Fig. 1a. The ratio $E / J$ tends to a finite value for small $J$, corresponding to the linear resistivity $\rho_{L}=\lim _{J \rightarrow 0} E / J$, which is nonzero but depends strongly on the temperature. This can be seen in Fig. 1b, where the linear resistivity shows an exponential decrease with temperature. In Fig. 1a, there is also a smooth crossover to nonlinear behavior that appears at smaller currents for decreasing temperatures. These are the main features expected for a $T=0$ superconducting transition [14, 15]. If the transition only occurs at $T=0$, then the correlation length should diverge for decreasing temperature and a temperature-dependent crossover is expected. The linear resistivity $\rho_{L}$ is finite at any nonzero temperature but thermally activated, $\rho_{L} \propto \exp \left(-E_{b} / k T\right)$, where $E_{b}$ is an energy barrier. If one assumes that the correlation length diverges as a power-law $\xi \propto T^{-\nu}$, then the behavior of the nonlinear resistivity normalized to $\rho_{L}$ can be cast in to the scaling form [14]

$$
\frac{E}{J \rho_{L}}=g\left(\frac{J}{T^{1+\nu}}\right)
$$

in $d=2$ dimensions, where $g$ is a scaling function with $g(0)=1$. A crossover from linear behavior, when $g(x) \sim 1$, to nonlinear behavior, when $g(x)>1$, should occur when $x \sim 1$. This leads to a characteristic current density $J_{n l}$ at which nonlinear behavior sets in that decreases with temperature as a power law $J_{n l} \propto T^{1+\nu}$. We now proceed to very the scaling hypothesis and obtain a numerical estimate of the critical exponent $\nu$. Fig. 2a shows a scaling plot according to Eq. 2 obtained by adjusting the single parameter $\nu$ so that a best data collapse is obtained. The dada collapse supports the scaling behavior and provides an estimate of $\nu=0.95$. An alternative estimate of $\nu$ can also be obtained from the crossover current density $J_{n l}$. Fig. $2 \mathrm{~b}$ shows the temperature dependence of $J_{n l}$ defined as the value of $J$ where $E / J \rho_{L}$ starts to deviate from a fixed value, chosen to be 2 , using the data in Fig. 1a. The behavior is consistent with a power-law behavior $J_{n l} \propto T^{1+\nu}$ and gives $\nu=1.07(8)$. Combining the two estimates gives $\nu=1.01(8)$. These results, including the numerical estimate of $\nu$, are in good agreement with previous calculations [10] using Langevin dynamics in the phase representation and MC dynamics in the vortex representation. 
(a)

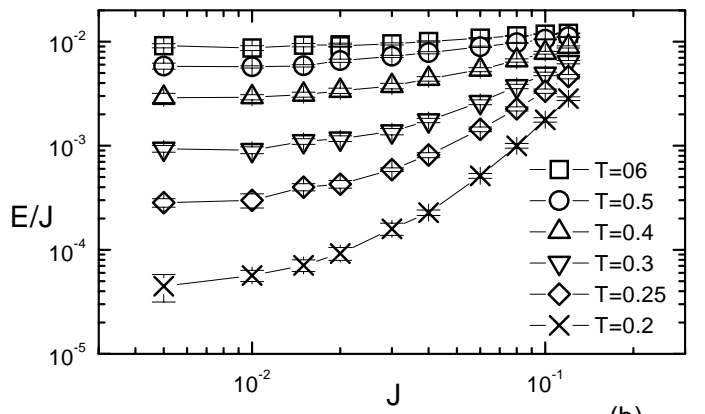

(b)

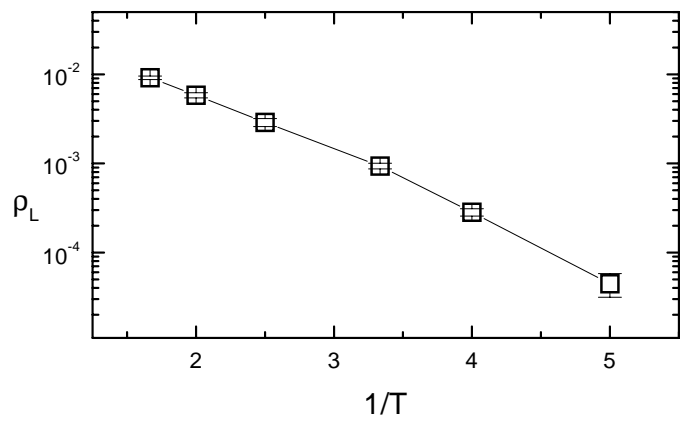

Figure 1. (a) Nonlinear resistivity $E / J$ for different temperatures $T$ in two dimensions, for a system size $L=34$; (b) Arrhenius plot for the temperature dependence of the linear resistivity $\rho_{L}$.

(a)
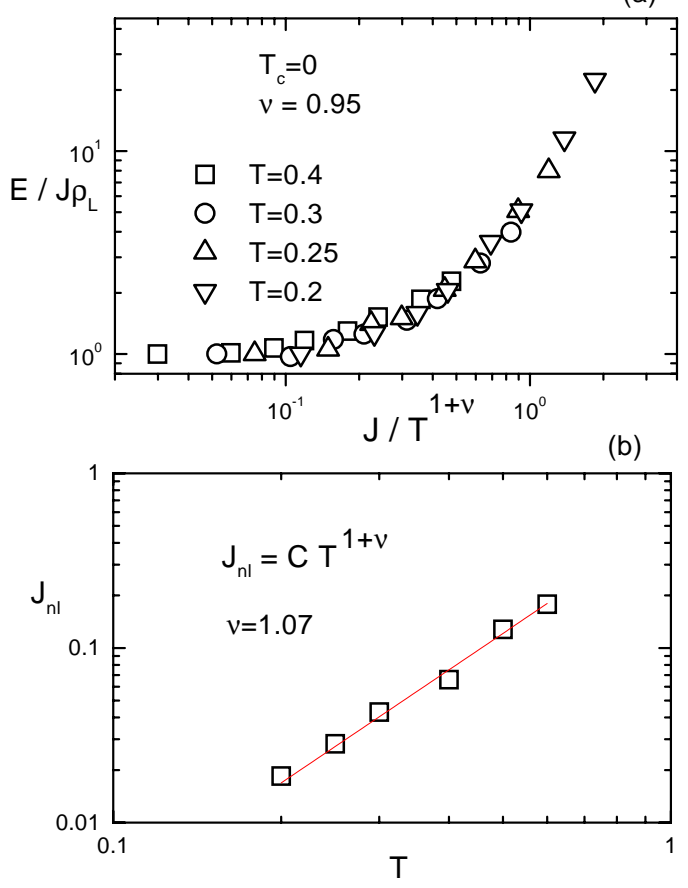

Figure 2. (a) Scaling plot $E / J \rho_{L} \times J / T^{1+\nu}$ for data in Fig. 1a with $\nu=0.95$; (b) Crossover current density $J_{n l}$ as a function of temperature from Fig. 1. (a)

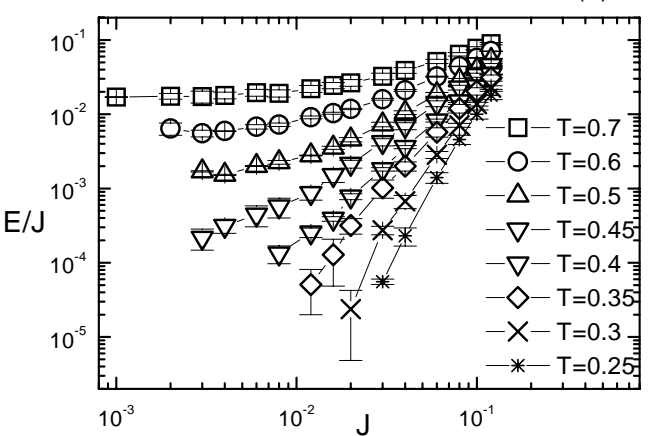

(b)

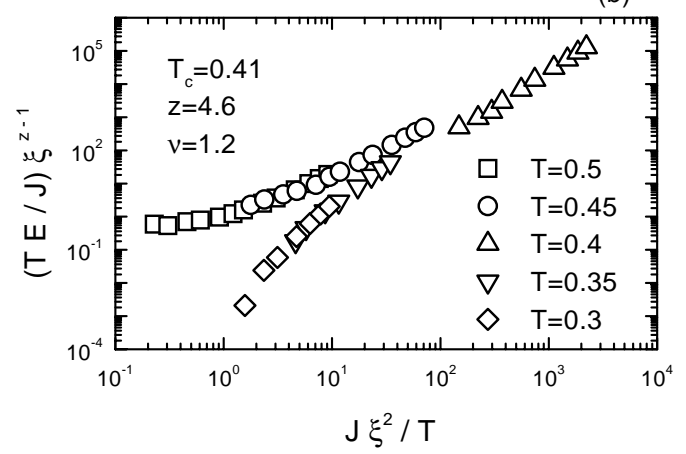

Figure 3. (a) Nonlinear resistivity $E / J$ for different temperatures $T$ in three dimensions, for system size $L=12$; (b) Scaling plot of the data near the transition and for small currents, with $\xi \propto\left|T / T_{c}-1\right|^{-\nu}$.

In three dimensions, the resistivity behavior is quite different, as shown in Fig. 3a, for a large system size $L=12$. The behavior is consisting with a resistive transition at an apparent critical temperature in the range $T_{c} \sim$ $0.3-0.45$. At higher temperatures, the linear resistivity $\rho_{L}=\lim _{J \rightarrow 0} E / J$ is finite while at lower temperatures, it extrapolates to zero. The phase transition can be confirmed by a scaling analysis of the nonlinear resistivity which assumes the existence of a continuous equilibrium transition at a finite temperature [14]. Near the transition, measurable quantities scale with the diverging correlation length $\xi \propto\left|T-T_{c}\right|^{-\nu}$ and relaxation time $\tau \propto \xi^{z}$, where $\nu$ and $z$ are the correlation-length and dynamical critical exponents, respectively. The nonlinear resistivity should then satisfy the scaling form [14]

$$
T E \xi^{z-1} / J=g_{ \pm}\left(J \xi^{2} / T\right)
$$

in $d=3$ dimensions where $g(x)$ is a scaling function. The + and - signs correspond to $T>T_{c}$ and $T<T_{c}$, respectively. A scaling plot according to this equation can then be used to verify the scaling arguments and the assumption of an underlying equilibrium transition at $J=0$. The optimal data collapse provides an estimate of $T_{c}$ and critical exponents. Such scaling plot is shown in Fig. 3b, obtained by adjusting the unknown parameters, giving the estimates $T_{c}=0.41(2), z=4.6(3)$ and $\nu=1.2(2)$. The values of $T_{c}, z$ and $\nu$ obtained by this scaling analysis using MC dynamics agree well with the previous estimates using the 
RSJ model [12] for the dynamics, clearly showing the existence of a phase-coherence transition at finite temperatures and also showing that the dynamic exponent $z$ is essentially the same. Our estimate of $T_{c}$ from the resistivity scaling is in good agreement with recent estimate of the critical temperature, $T_{c h}=0.38-0.41$, for the chiral-glass transition from MC simulations [7]. The agreement is quite intriguing since it apparently supports the suggestion [12] that chirality and phase variables may order simultaneously. Nevertheless, this phase-coherence transition is in sharp contrast with MC simulations of the phase-overlap distribution function $[6,7]$. On the other hand, a phase-coherence transition at $T>0$, is consistent with calculations of the spin stiffness exponent in the ground state showing that the lower-critical dimension for spin order in the XY-spin glass model [9] is below 3 which implies that a phase-coherence transition at finite temperatures is possible in three dimensions. More recent, improved calculations in the vortex representation, also clearly shows a large and positive stiffness exponent [16]. In addition, calculations of the linear resistivity $\rho_{L}$ (zero current bias) from MC dynamics simulations in the vortex representation [11], shows an equilibrium resistive transition. The estimate of the static exponent $\nu$ agrees with the present estimate from the nonlinear resistivity but the dynamic exponent [11] $z=3.1$ is significantly lower. Surprisingly enough, our calculations of $z$ show the same result for the MC and RSJ dynamics. Additional calculations using Langevin dynamics with onsite dissipation of the grains in the phase representation give the same result [17]. In spite of that, it is possible that the different $z$ is a result of the particular dynamics in the vortex representation. In fact, vortex variables are collective excitations in the phase representation and thus lead to long-range correlations for the phases, suggesting that these representations may belong to different dynamic universality classes.

In conclusion, our driven MC dynamics simulations of the resistivity scaling in $\pi$ junction granular superconductors, shows that, in two dimensions, the linear resistivity is nonzero at finite temperatures and the behavior is consistent with a phase transition at zero temperature in good agreement with previous calculations [10]. In three dimensions, we find a resistive transition at finite temperatures in the chiral glass phase and determine the corresponding critical exponents. This transition should be observable in nonlinear resistivity measurements in bulk samples. In fact, it is consistent with resistivity measurements in ceramic $\mathrm{YBa}_{2} \mathrm{Cu}_{4} \mathrm{O}_{8}$ bulk samples [19] near the onset of the para- magnetic Meissner effect. The power-law exponent $\alpha$ for the nonlinear contribution found in these measurements can be related [18] to the dynamic critical exponent $z$ of the transition.

This work was supported in part by computer facilities from CENAPAD and FAPESP (grant nos. 03/00541-0 and 01/03100-9).

\section{References}

[1] M. Sigrist and T.M. Rice, Rev. Mod. Phys. 67, 503 (1995).

[2] C.C. Tsuei and J.R. Krtley, Rev. Mod. Phys. 72, 969 (2000).

[3] H. Hilgenkamp et al., Nature 422, 50 (2003).

[4] F.V. Kusmartsev, Phys. Rev. Lett. 69, 2268 (1992).

[5] A. Gardchareon, R. Mathieu, P.E. Jônsson, and P. Nordblad, Phys. Rev. B 67, 052505 (2003).

[6] H. Kawamura, J. Phys. Soc. Jpn 64, 711 (1995).

[7] H. Kawamura and M.S. Li, Phys. Rev. Lett. 87, 187204 (2001); Phys. Rev. Lett. 78, 1556 (1997); Phys. Rev. B 54, 619 (1996).

[8] W. Braunisch, N. Kanauf, V. Kataev, S. Neuhausen, A. Grütz, A. Kock, B. Roden, D. Khomskii, and D. Wohleben, Phys. Rev. Lett. 68, 1908 (1992).

[9] J. Maucourt and D.R. Grempel, Phys. Rev. Lett. 88, 770 (1998).

[10] E. Granato, Phys. Rev. B58 (1998) 11161; B61, 391 (2000).

[11] C. Wengel and A.P. Young, Phys. Rev. B 56, 5918 (1997).

[12] E. Granato, J. Magn. Magn. Matter. 226, 366 (2001).

[13] W. M. Saslow, M. Gabay, and W.-M. Zhang, Phys. Rev. Lett. 68, 3627 (1992).

[14] D.S. Fisher, M.P.A. Fisher, and D.A. Huse, Phys. Rev. B 43, 130 (1991); R.A. Hyman, M. Wallin, M.P.A. Fisher, S.M. Girvin, and A.P. Young, Phys. Rev. B 51, 15304 (1995).

[15] E. Granato, in "Studies of high-temperature superconductors", Ed. A. Narlikar (Nova Science Publishers, New York, 2001), vol. 39, p. 149.

[16] J.M. Kosterlitz and N. Akino, Phys. Rev. Lett. 82, 4094 (1999).

[17] E. Granato, Physica C, to be published.

[18] E. Granato, unpublished.

[19] T. Yamao, M. Haiwara, K. Koyama, and M. Matsuura, J. Phys. Soc. Jpn. 68, 871 (1999). 